\title{
Operational Flexibility of Small-Scale Electricity-Coupled Heat Generating Units
}

\author{
Anke Weidlich $^{1}$ (1) . Ahsan Zaidi ${ }^{2}$ \\ Received: 4 September 2018 / Accepted: 16 April 2019 / Published online: 29 April 2019 \\ (C) Springer Nature Singapore Pte Ltd. 2019
}

\begin{abstract}
Heat generation that is coupled with electricity usage, like combined heat and power generators or heat pumps, can provide operational flexibility to the electricity sector. In order to make use of this in an optimized way, the flexibility that can be provided by such plants needs to be properly quantified. This paper proposes a method for quantifying the flexibility provided through a cluster of such heat generators. It takes into account minimum operational time and minimum down-time of heat generating units. Flexibility is defined here as the time period over which plant operation can be either delayed or forced into operation, thus providing upward or downward regulation to the power system on demand. Results for one case study show that a cluster of several smaller heat generation units does not provide much more delayed operation flexibility than one large unit with the same power, while it more than doubles the forced operation flexibility. Considering minimum operational time and minimum down-time of the units considerably limits the available forced and delayed operation flexibility, especially in the case of one large unit.
\end{abstract}

Keywords Operational flexibility · Electricity-coupled heat generation · Combined heat and power generation . Heat pumps - Virtual power plants $\cdot$ Smart grid

\section{Nomenclature}

$\begin{array}{ll}\text { Abbreviations } \\ \text { CHP } & \text { Combined heat and power } \\ \text { DH } & \text { District heating } \\ \text { EHG } & \text { Electricity-coupled heat generator } \\ \text { HP } & \text { Heat pump } \\ \text { TES } & \text { Thermal energy storage } \\ \text { VPP } & \text { Virtual power plant }\end{array}$

\section{Superscripts}

Max Symbol related to the maximum operation mode

Min Symbol related to the minimum operation mode Subscripts

$t \quad$ Denotes the current time interval, $t \in[0,1, \ldots, T]$

Anke Weidlich

anke.weidlich@inatech.uni-freiburg.de

1 Department of Sustainable Systems Engineering, University of Freiburg, Freiburg, Germany

2 Institute of Energy Systems Technology, University of Applied Sciences Offenburg, Offenburg, Germany

\begin{tabular}{|c|c|}
\hline down & Shut-down \\
\hline DT & Down-time \\
\hline OT & Operating time \\
\hline up & Start-up \\
\hline \multicolumn{2}{|l|}{ Symbols } \\
\hline$\Delta P_{\text {forced }}$ & Power flexibility to forceable from EHG (W) \\
\hline$\Delta t$ & Time interval (here: $15 \mathrm{~min}$ ) \\
\hline$\Delta_{\text {delayed }}$ & Flexibility to delay EHG operation (h) \\
\hline$\Delta P_{\text {forced }}$ & Flexibility to force EHG operation (h) \\
\hline $\bar{E}_{\mathrm{TES}}$ & Maximum capacity of energy storage (Wh) \\
\hline $\bar{n}_{\text {up }, \mathrm{DT}}$ & $\begin{array}{l}\text { Maximum number of units to start up due to } \\
\text { minimum down-time constraint }\end{array}$ \\
\hline $\bar{n}_{\text {up }, \text { OT }}$ & $\begin{array}{l}\text { Maximum number of units to start up due to } \\
\text { minimum operation time constraint }\end{array}$ \\
\hline $\bar{P}_{\mathrm{EHG}}$ & Rated thermal power of one EHG unit (W) \\
\hline Max & Value of the upper boundary function (Wh) \\
\hline Min & Value of the lower boundary function (Wh) \\
\hline$n$ & Minimum number of units to operate \\
\hline $\bar{d}$ & Minimum down-time (number of time intervals) \\
\hline$E_{\mathrm{TES}}$ & $\begin{array}{l}\text { Energy in the storage at the end of a time interval } \\
\text { (Wh) }\end{array}$ \\
\hline$k$ & Total number of EHG units \\
\hline$n$ & Number of EHG units operating at time $t$ \\
\hline$n^{*}$ & Adjusted number of EHG units operating \\
\hline
\end{tabular}




$\begin{array}{ll}n_{\text {down }} & \begin{array}{l}\text { Number of units shut down at the beginning of a } \\ \text { time interval }\end{array} \\ n_{\text {up }} & \begin{array}{l}\text { Number of units newly started up at the } \\ \text { beginning of a time interval }\end{array} \\ P_{\text {Boiler }} & \begin{array}{l}\text { Boiler power output }(\mathrm{W}) \\ Q\end{array} \\ r & \begin{array}{l}\text { Heat demand (Wh) } \\ \text { Minimum operation time (number of time }\end{array} \\ T & \begin{array}{l}\text { intervals) } \\ \text { Time horizon (set of all times } t \text { considered) }\end{array}\end{array}$

Introduction

The transition towards more sustainable energy systems drives the increased use of renewable energy sources as a substitution for fossil energy usage. The resulting growing number of energy conversion installations based on fluctuating renewable energy sources increases the variability of power generation. As the phase-out of dispatchable generation is simultaneously promoted in many countries, there is an increasing value for flexibility provided by other components in the power system, in order to match demand with supply at all times [28]. Simultaneously, most of the options available for substituting fossil energy in the supply of all energy services involve an increased electrification, especially for the provision of heat or mobility. This creates new challenges to, but can also provide solutions for power systems.

New flexibility options have been extensively discussed in the literature, and a few of them are operational in some energy systems; [9] for example provides a good overview of flexibility from distributed energy resources and their possible integration into electricity markets. Demand response (DR) as one example offers a large technical potential [13], but its actual implementation depends on regulations and specific energy system characteristics. As a consequence, the technical potential of demand response is not exploited to its full extent in many countries [25]. Another possible source of flexibility is to link electricity flows with other energy systems, specifically with the heat sector [30]. The growing usage of both heat pumps and decentralized combined heat and power (CHP) plants increasingly couples heat provision with the electricity system: small-scale CHP plants deliver electricity while generating heat, whereas heat pumps consume electricity while providing heat. For this reason, we collectively refer to these plants as electricity-coupled heat generators (EHG), and characterize the flexibility that these units can provide to the power system.

This research is based on the hypothesis that EHG can provide flexibility to the electricity system, by allowing some degree of freedom in the timing of their operation. Moreover, it is expected that if plants are operated in on/off mode (i. e. at either $0 \%$ or $100 \%$ of their rated power), flexibility is increased if an aggregation of several smaller units are operated as opposed to one large unit of the same power. It can also be expected that operational constraints such as minimum operation and minimum down-time reduce the available flexibility of EHG clusters, and even more so for large single units. The proposed model allows for probing these conjectures, all under the assumption of perfect knowledge of the heat demand to be served by the EHG plants (i. e. no uncertainty is considered here).

\section{Related Work}

At the more general level, there are several papers that define operation flexibility for power systems, and suggest metrics for quantifying this flexibility. To mention a few, the National Renewable Energy Laboratory NREL defines flexibility simply as the ability of a power system to respond to change in demand and supply [5]. They define several metrics to measure flexibility, such as the maximum upward or downward change in the supply/demand balance that a power system is capable of meeting over a given time horizon and a given initial operating state. Similarly, Ulbig and Andersson propose a generic framework for quantifying flexibility through the three metrics of ramp capability (in $\mathrm{MW} / \mathrm{min}$ ), power capacity (in MW), and energy (in MWh) [29]. Ma et al. and Nosair and Bouffard provide guidance on flexibility planning in power systems $[17,19]$. Alizadeh et al. provide an extensive literature survey on various aspects of flexibility for energy systems with high shares of variable renewable energy [1]. A review of research results on the analysis of flexibility demand for high VRE power systems is given by Kondziella and Bruckner [15].

There are a number of papers that describe and quantify more specifically the flexibility that buildings, and especially their heating systems and hot water appliances in buildings can deliver. Lopes et al. provide an overview of many existing approaches, and distinguish two categories of flexibility in buildings, namely the thermal energy storageassisted energy flexibility and the flexibility obtained from operation shifting [16]. In the former category, the energy flexibility is usually assessed based on an anticipated demand at short sight, taking into account the thermal comfort needs of the building users. In another more recent review, Reynders et al. compare and evaluate existing definitions and quantification methodologies for flexibility in buildings [23]. They distinguish the two classes of direct and indirect approaches. Direct approaches predict a building's energy flexibility in a bottom-up manner, while indirect approaches are based on past data and assume a specific energy system.

Reynders et al. assume a minimum comfort temperature that they use as a reference for calculating the active flexibility potential from a thermal appliance based on 
forecast energy demand [22]. D'Hulst et al. quantify the flexibility offered by domestic electrical devices including electric hot water buffers [7]. They describe flexibility as the potential to increase or decrease power consumption as a function of the time of the day, along with the possible duration of such power change. They find that a maximum of $2.4 \mathrm{~kW}$ in negative flexibility (increased load) can be realized per buffer of 2001 volume, while only $0.3 \mathrm{~kW}$ of positive flexibility (load decrease) per buffer is obtainable.

In other research approaches, the economic aspect of flexibility is studied in detail. Pedersen et al. [21] develop an aggregated model with the main aim of obtaining increased flexibility for trading in the intra-day market. The results from this model serve as a reference for purchase of energy on the day-ahead market. The authors propose a scheduling algorithm for an efficient distribution of purchased energy among several houses in a cluster, considering their current temperature levels, minimum heat pump on/off time, reference temperature, and run time status.

De Coninck and Helsen present focus on the flexibility provided by heating systems, using thermal properties of buildings [6]. They simulate different control strategies and derive flexibility cost curves from the solutions obtained by these optimal control problems. The cost curve, thus, links the amount of available flexibility at a given time to its activation cost.

Finck et al. also use optimal control for determining demand flexibility provided by heat pumps, electric heaters and thermal energy storage tanks for the case of office buildings [10]. They quantify (demand) flexibility through different indicators that account for energy, power and costs. In addition, they introduce the instantaneous power flexibility as another flexibility indicator, which they quantify for the cases of thermal storage charging, discharging or idle mode.

Nuytten et al. [20] (and similarly Six et al. [26]) study the flexibility provided by one CHP generation unit with thermal energy storage. For this, they use minimum and maximum operation curves which set boundaries to possible EHG operation. This allows measuring the possible duration of forced and delayed operation of the plant.

Stinner et al. discuss three types of flexibility that are offered by electricity-coupled heat generation plants with thermal energy storage, namely temporal flexibility (for which they refer to [20]), power flexibility, and energy flexibility [27]. They quantify the power that a heat generator could deliver or consume (CHP plants and for heat pumps or heating rods, respectively) as the difference between a maximum power and the power that would be provided/consumed in a reference case without flexibility usage.

Dimeas et al. discuss the use of flexibility from buildings in different use cases. The suggested approaches, which are tested in the field, comprise shifting electric hot water production in a microgrid setting on the one hand, and market participation of heat pumps, CHP units, and other electric devices on the other hand [8]. Similar approaches are presented in other studies on microgrid management, e. g. [2, 4], which determine optimized operational schedules. Determining flexibility as feasible deviations from a previously determined optimal schedule is another approach for flexibility quantification found in the literature. For example, [31] optimize charging cycles for electric vehicles and formulate bids for flexibility based on feasible delayed or advanced charging compared to scheduled charging.

Kohlhepp and Hagenmeyer model EHG as storage units, so that flexibility can be quantified in analogy to a generic energy storage unit. They consider the entire thermally usable building mass as storage, and define its storage capacity as the electrical work corresponding to the thermal energy to completely fill or empty the storage, i. e. to heat up or cool down the building within previously defined bounds [14].

Most papers focus on the flexibility assessment of single CHP or HP units. Among the few who consider pools of several units, Fischer et al. models a larger pool of heat pumps in residential buildings [11]. The authors simulate the response of the heat pumps to a trigger signal that represents a request for flexibility (i. e. to turn heat pumps on or off). They characterize flexibility by the maximum power change as a response to the trigger signal, and by shiftable energy and regeneration time. The duration of possible on or off operation on request, however, is not quantified.

Another approach for modeling flexibility is proposed by Förderer et al. [12]. They argue that a model that can be used for creating feasible load profiles of a distributed energy resource represents a potential model for the flexibility of that particular resource. They use artificial neural networks as surrogate models for the flexibility definition. Through this, they avoid the burden of explicitly modeling individual plant types. A similar approach has previously been proposed by MacDougall et al. [18], who use both artificial neural networks and multivariate linear regression to determine the aggregate flexibility of a virtual power plant consisting of many small heating devices.

\section{Contribution of This Work}

Most previous work focuses on characterizing single flexibility units. We specifically focus on clusters of several EHG units that are operated under a common control strategy as a virtual power plant (VPP) [3, 24], for example within a local heat network. As a consequence, the aggregated flexibility of the cluster can be compared to that of single units, thus quantifying the value of virtual clusters. 
In addition, minimum operation time and minimum downtime of the individual EHG units are explicitly accounted for, as these are relevant restrictions for plant operation which can severely limit the available flexibility potential.

A method for defining and quantifying the flexibility provided through EHG generation (CHP plants as electricity generators or heat pumps as electricity consumers) along with thermal energy storage capacity is proposed. The focus is on small-scale EHG systems, which are used for serving the heat demand of individual buildings or small clusters of buildings. Building on the basic approach of [20], the flexibility for the EHG cluster is quantified as the time period over which the operation of the units can be influenced, i. e. either forced (positive flexibility in case of CHP, negative flexibility in case of HP) or delayed (vice versa). At each time step, the maximum flexibility is calculated, assuming that enough lead time is given to prepare EHG generation such that the necessary initial state for the desired flexibility potential is reached.

Different from other approaches for quantifying flexibility, such as [27, 31], no (optimal) reference schedule needs to be determined for the flexibility calculation, but any operation between two extreme (minimum and maximum) schedules is possible. The calculation of this set of feasible schedules, or operational zone as we refer to it here, does not require computationally expensive machine learning approaches as used in $[12,18]$. At the same time, it does not require a high level of detail about the modeled units, which makes it easy to implement for new units in practical applications.

The proposed model was implemented in Matlab and applied to the case of a CHP cluster situated in Germany. Results from this case study are presented and discussed. The remainder of the paper is structured as follows: "Methodology" describes the method for flexibility assessment developed in this work, and "Case Study and Results" presents the results from a case study where this method has been applied. Section "Discussion" discusses the results obtained, and "Conclusion" finally concludes.

\section{Methodology}

The proposed method for defining flexibility of electricitycoupled heat generators assumes that the plants are always operated in combination with a thermal energy storage (TES) unit, and that heat can be served either directly from the output of the EGH unit or from the thermal storage. The methodology builds on [20], but extends the approach by incorporating the aspects of minimum downtime and minimum operation time, as these are very relevant limitations of small-scale EHG units operation in practice. In addition, the model does not only consider single units, but can account for clusters of $k$ (identical) EGH units.

In the given approach, flexibility occurs between a Minimum curve and a Maximum curve. These curves provide a range between which the operation of a EHG unit is possible. This range is referred to as the operational zone, visualized as the gray area in Fig. 1, which shows the Min and Max curves, respectively, as the lower and upper boundaries of the operational zone. The formulation of the curves is further detailed in the following sections.

\section{Lower Operational Boundary}

Following the idea of [20], the minimum curve $\operatorname{Min}_{t}$ is formulated such that it represents the lower bound of possible EHG operation. It can be thought of as a theoretical operational mode in which the EHG operation is delayed as much as possible, within the boundaries of serving the demand. An EHG unit should only run when heat the demand of the next time interval cannot be met by the content of the storage unit, and the TES is kept at a minimum charge. In the following, all variables related to this minimum operation mode are denoted by the upper index Min.

In a cluster of $k$ identical EHG units with individual rated power $\bar{P}_{\text {EHG }}$, it is assumed that the units can only run at full power or be off, with no power modulation possible. This is consistent with actual designs of many smallscale decentralized EHG systems. As different numbers of units $n_{t}^{\text {Min }}$ can be operational at each time interval $t$, some step-wise modulation possibility results for the cluster. Minimum operation time and minimum down time is accounted for in the construction of the Min curve, as these represent considerable limitations of flexible operation in practice.

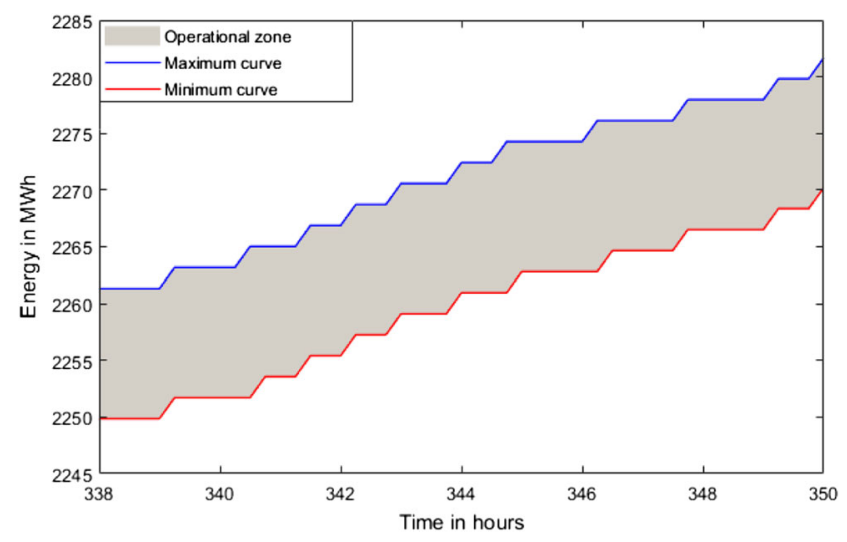

Fig. 1 Min and max curves and EHG operational zone 
The minimum curve is defined as the cumulative sum of energy entering into the system such that the heat demand $Q_{t}$ is met at all times $t$ while keeping the storage level as low as possible. The (thermal) power delivered by the EHG cluster, with $n_{t}^{\mathrm{Min}}$ units operating during the whole time interval $t$ in the minimum curve mode, is given as the product $n_{t}^{\mathrm{Min}} \cdot \overline{\mathrm{P}}_{\mathrm{EHG}}$. The determination of $n_{t}^{\mathrm{Min}} \in[1, \ldots, k]$ is described in "Number of EHG Units Operating".

Any excess heat provided by the EHG units is stored in the TES. Heat from the TES can then be used for serving demand at later time steps. The storage charge level at time $t, E_{\mathrm{TES}, t}^{\mathrm{Min}}$ is given by Eq. 1, starting from an initial charge level $E_{\mathrm{TES}, 0}^{\mathrm{Min}}$.

$E_{\mathrm{TES}, t}^{\mathrm{Min}}=E_{\mathrm{TES}, t-1}^{\mathrm{Min}}+n_{t}^{\mathrm{Min}} \cdot \bar{P}_{\mathrm{EHG}} \cdot \Delta t-Q_{t}$

\section{Number of EHG Units Operating}

Several constraints need to be considered when determining $n_{t}^{\mathrm{Min}}$. The first one reflects the need to meet the heat demand of the consumer, and that with the lowest possible number of EHG units operating. This is specified in Eq. 2. The capacity of the TES is denoted as $\bar{E}_{\text {TES }}$ and must never be exceeded. As heat from EHG operation cannot be dumped, generation must stop when excess heat cannot be accommodated by the thermal energy storage.

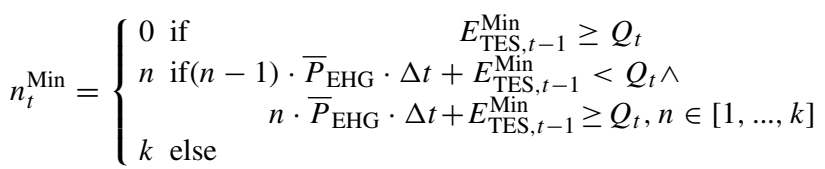

In the latter of the three cases in Eq. 2, a peak boiler will operate for satisfying the remaining heat demand that cannot be served by the EHG units and the storage unit together.

\section{Down-Time and Operational Time Constraints}

The number of EHG units operating in $t$ might have to be reduced, if minimum operation or minimum down-time constraints apply. Operation of a new unit is only allowed after a minimum number $d$ of time intervals has elapsed since their last shut-down. Equivalently, all units once turned on must operate a minimum number $r$ of time steps before they can be shut down again. This represents technical constraints given by many plant manufacturers, that should reduce wearing from too many switching operations. Given that the minimum down-time and operation time constraints apply to the Min curve in the same way as for the Max curve, the superscript Min is omitted in the following descriptions.

The number of units that can be switched on or off in time period $t$ is, thus, dependent on the number of units operating in the past time intervals, and the number of units that can operate in the coming time intervals. When the number of EHG units $n_{t}^{\mathrm{Min}}$ needed in $t$ is higher than that of the previous interval, $n_{t-1}^{\mathrm{Min}}$, then $n_{\mathrm{up}, t}^{\mathrm{Min}}$ additional units must be switched on at the beginning of that time interval. The number of units newly switched on is given by

$n_{\text {up }, t}=\max \left(n_{t}-n_{t-1} ; 0\right)$

Equivalently, the number of units that are switched off at the beginning of one time interval, $n_{\text {down }, t}$, is given by

$n_{\text {down }, t}=\max \left(n_{t-1}-n_{t} ; 0\right)$

The maximum number of units available for start-up at the beginning of $t$ when considering minimum down-time (DT), $\bar{n}_{\mathrm{up}, \mathrm{DT}, t}$, is the remaining units not yet in operation, reduced by the units that were shut down too recently, i. e. in the last $d$ time steps (5). As all units are identical, only the overall number of units needs to be considered.

$\bar{n}_{\text {up }, \mathrm{DT}, t}=k-n_{t-1}-\sum_{\tau=1}^{d-1} n_{\text {down }, t-\tau}$

At the same time, it must be ensured that all excess heat from EHG operation can be stored in the buffer. As units newly switched on cannot be shut down before having run the minimum operation time (OT) of $r$ time intervals, compliance with the storage constraint must also be checked before start-up, as defined in Eq. 6 (following the same reasoning as in Eq. 2).

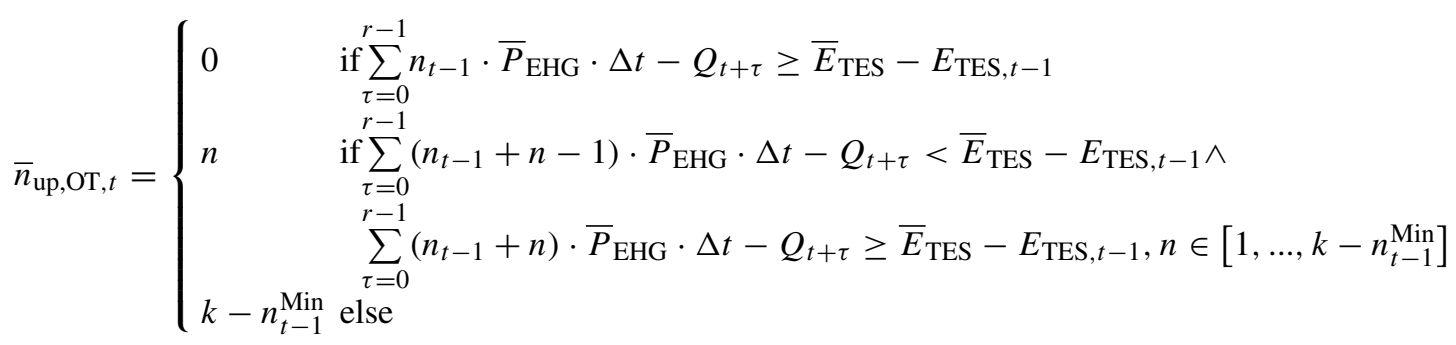


The minimum operation time constraint may also set a lower limit $\underline{n}_{t}$ to the number of plants operating in $t$, as a unit that has been newly switched on cannot be shut down before having operated $r$ intervals (7).

$\underline{n}_{t}=\sum_{\tau=1}^{r-1} n_{\mathrm{up}, t-\tau}$

If either one of the allowed numbers of units to be switched on $\bar{n}_{\mathrm{up}, \mathrm{DT}, t}^{\mathrm{Min}}$ or $\bar{n}_{\mathrm{up}, \mathrm{OT}, t}^{\mathrm{Min}}$, is lower than the desired number $n_{\mathrm{up}, t}^{\mathrm{Min}}$, then $n_{t}^{\mathrm{Min}}$ needs to be adjusted for avoiding violation of the minimum down-time and operation time constraints. Simultaneously, an adjustment might become necessary if one or several units should be switched off, but have not yet been operating the required $r$ time steps, as defined in Eq. 7. The adjusted number of operating units, $n_{t}^{\mathrm{Min} *}$, is given by Eq. 8 .

$$
\begin{gathered}
n_{t}^{\mathrm{Min} *}=\max \left(n_{t}^{\mathrm{Min}}-\max \left(n_{\mathrm{up}, t}^{\mathrm{Min}}-\bar{n}_{\mathrm{up}, \mathrm{DT}, t}^{\mathrm{Min}} ; n_{\mathrm{up}, t}^{\mathrm{Min}}\right.\right. \\
\left.\left.-\bar{n}_{\mathrm{up}, \mathrm{OT}, t}^{\mathrm{Min}} ; 0\right) ; \underline{n}_{t}^{\operatorname{Min}}\right)
\end{gathered}
$$

The procedure of determining $n_{t}^{\text {Min }}$ and $n_{t}^{\text {Min* }}$ is visualized in Fig. 26 in the Appendix.

\section{Formulation of the Minimum Curve}

The last element of the Min curve is the definition of any auxiliary energy delivered to the system. If demand exceeds the amount of thermal energy delivered by the currently operating EHG units and the TES discharge, a peak boiler steps in for serving any remaining demand. It is assumed that the boiler can always meet the entire heat demand $Q_{t}$ of the consumer. Boiler operation in the minimum operation mode is specified as:

$P_{\mathrm{Boiler}, t}^{\mathrm{Min}}=\max \left(\frac{Q_{t}-E_{\mathrm{TES}, t-1}^{\mathrm{Min}}}{\Delta t}-n_{t} \cdot \bar{P}_{\mathrm{EHG}} ; 0\right)$

The Min curve is now described as follows:

$$
\operatorname{Min}_{t+1}=\operatorname{Min}_{t}+\left(n_{t}^{\mathrm{Min} *} \cdot \bar{P}_{\mathrm{EHG}}+P_{\mathrm{Boiler}, t}^{\mathrm{Min}}\right) \cdot \Delta t ; \operatorname{Min}_{0}=0
$$

\section{Upper Operational Boundary}

In the same way as the lower boundary, a maximum curve is calculated as the upper boundary of possible EHG operation. It follows the objective to operate the plant(s) as early as possible, thus keeping the thermal storage at the highest possible charge level while meeting the heat demand. The maximum curve is also defined as the cumulative energy amount entering into the system.

The main limitation in the maximum mode is set by the storage capacity $\bar{E}_{\text {TES }}$. The update of the current storage level in the maximum mode is the same as in the minimum mode (1). In analogy to Eq. 2, the required number of units operating in the maximum mode, $n_{t}^{\text {Max }}$ is defined in Eq. 11. It reflects the goal to meet the demand with the highest possible number of EHG units operating, without surpassing the buffer storage capacity $\bar{E}_{\text {TES }}$.

$n_{t}^{\mathrm{Max}}=\left\{\begin{array}{l}n \text { if } \quad n \cdot \bar{P}_{\mathrm{EHG}} \cdot \Delta t-Q_{t} \leq \bar{E}_{\mathrm{TES}}-E_{\mathrm{TES}, t-1}^{\mathrm{Max}} \wedge \\ \quad(n+1) \cdot \bar{P}_{\mathrm{EHG}} \cdot \Delta t-Q_{t}>\bar{E}_{\mathrm{TES}}-E_{\mathrm{TES}, t-1}^{\mathrm{Max}}, n \in[0, \ldots, k-1] \\ k \text { else }\end{array}\right.$

Based on the number of units switched on or off in time $t, n_{\mathrm{up}, t}^{\mathrm{Max}}$ or $n_{\mathrm{down}, t}^{\mathrm{Max}}$, as defined in Eqs. 3 and 4 , the operational constraints defined in "Down-Time and Operational Time Constraints" apply similarly for the Max curve. This leads to the formulation of the adjusted number of units operating in time $t$ as follows:

$$
\begin{aligned}
n_{t}^{\mathrm{Max} *}=\max \left(n_{t}^{\mathrm{Max}}\right. & -\max \left(n_{\mathrm{up}, t}^{\mathrm{Max}}-\bar{n}_{\mathrm{up}, \mathrm{DT}, t}^{\mathrm{Max}} ; n_{\mathrm{up}, t}^{\mathrm{Max}}\right. \\
- & \left.\left.\bar{n}_{\mathrm{up}, \mathrm{OT}, t}^{\mathrm{Max}} ; 0\right) ; \underline{n}_{t}^{\mathrm{Max}}\right)
\end{aligned}
$$

The deployment of the peak boiler is defined as in the minimum operational mode, given in Eq. 9. With these specifications, the Max curve can be defined as the upper boundary for possible EHG operation as follows:

$$
\operatorname{Max}_{t+1}=\operatorname{Max}_{t}+\left(n_{t}^{\mathrm{Max} *} \cdot \bar{P}_{\mathrm{EHG}}+P_{\mathrm{Boile}, t}^{\mathrm{Max}}\right) \cdot \Delta t ; \operatorname{Max}_{0}=0
$$

\section{Temporal Flexibility Definition}

In the approach described here, flexibility is defined as the time period over which the generation of a cluster of EHG units can be flexibly controlled. From an electricity system view, it can sometimes be desirable to delay heat generation in order to avoid coupled electricity generation (in a CHP plant, e. g. during times of low or even negative electricity prices) or electricity consumption (in a heat pump, e. g. during peak demand times or while electricity prices are high). Equivalently, there can be a value for forcing heat generation to operate in the opposite situation for each of the heat generation types. The two types of flexibility of interest therefore quantify the amount of time (in hours) that operation can be forced or delayed on demand, while still using the CHP or HP units as primary heat generators (thus avoiding boiler operation) and while satisfying all 
constraints described in "Lower Operational Boundary" and "Upper Operational Boundary".

For the calculation of flexibility based on the Min and Max curves, we follow the same definition as given [20]. In the following, this approach is summarized for the maximum power that the heat generation units can provide, i. e. it quantifies how long no unit can operate (delayed case) or how long all units can simultaneously operate (forced case) at best. A more detailed discussion of allowing a subset of units to operate is presented in "Deployable Power During Flexibility Periods".

Flexibility to Delay Operation The maximum time for which the operation of an EHG can be postponed at a given time step $t, \Delta_{\text {delayed, } t}$, is found as the horizontal distance between the Max and Min curves. Starting on the Max curve value for time $t$, the corresponding time for which the Min curve has the same energy level as $\operatorname{Max}_{t}$, $t^{*}$, is found through linear interpolation between the two closest neighboring values. The flexibility value is given by the difference between this interpolated value $t^{*}$ and $t$, and reflects the period over which the demand can be satisfied through the thermal energy storage. The approach is visualized in Fig. 2 (at $t=505.5$ hours for the data set used in the case study described later).

Flexibility to Force Operation The maximum time $\Delta_{\text {forced }, t}$ for which all EHG units can be forced to operate on demand from time step $t$ onwards is given by another distance between the Min and Max curves. Starting on the Min curve, a linear function with the slope equal to the EHG power is put through $\mathrm{Min}_{t}$, and the point at which this function intersects with the Max curve denotes the time $t^{*}$ at which operation must stop. Again, the flexibility value is given by the difference between $t^{*}$ and $t$, and reflects the period over which the EHG can run without exceeding the TES

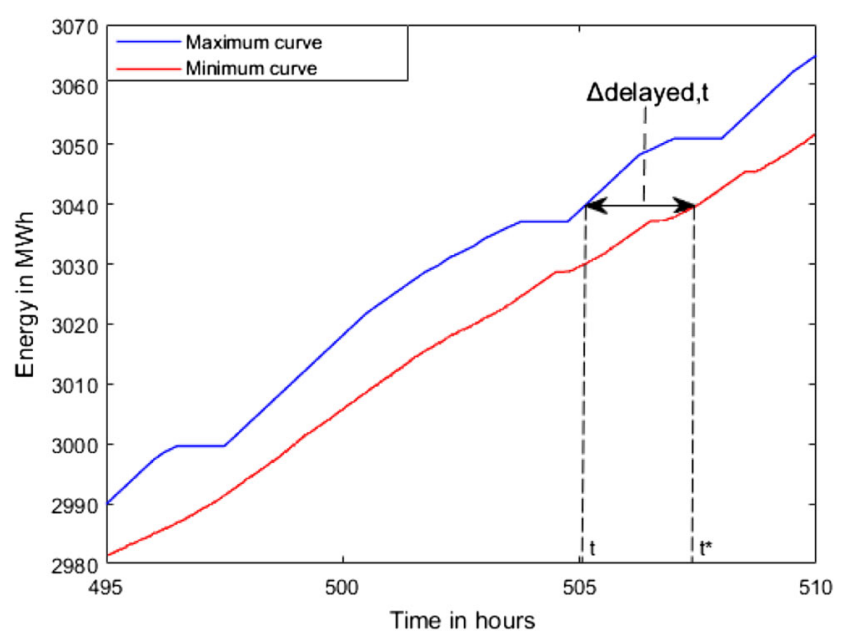

Fig. 2 Flexibility to delay operation

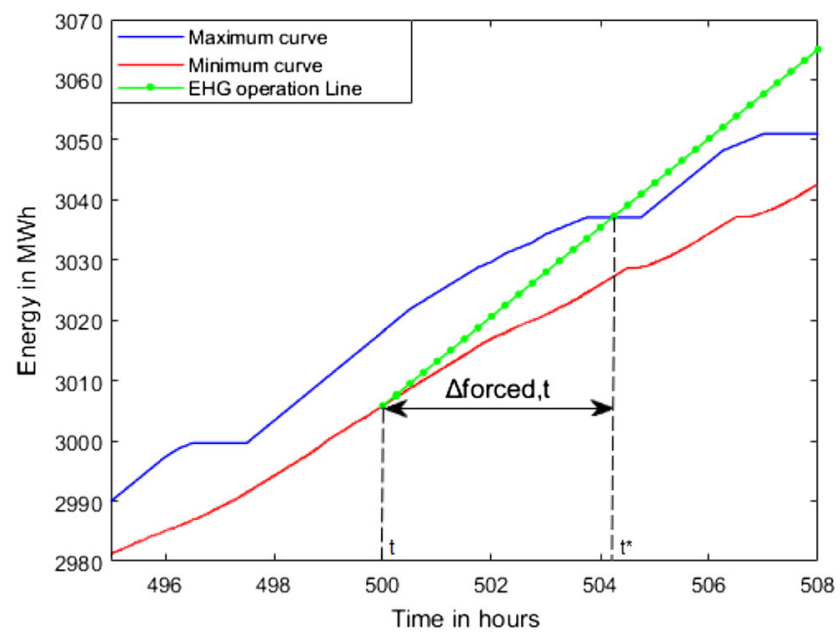

Fig. 3 Flexibility to force operation

charge capacity. This is visualized in Fig. 3 at $t=500$ hours.

Special Cases During periods of high heat demand, the Min and Max curves often coincide. In the example shown in Fig. 4, heat demand is higher than what the EHG units can serve at $t=3048 \mathrm{~h}$ and the following time periods, as the slope of EHG operation is lower than that of the Min and Max curves. Therefore, all heat generators have to operate. In this case, the forced operation flexibility is zero. This accounts for the fact that the EHG operation line is outside of the operational zone until it intersects again with the Min curve (at around $t=3058 \mathrm{~h}$ ).

When heat demand decreases for a short time after Min and Max curves being equal, and then increases again, this can result in the formation of small "flexibility pockets" as visualized in Fig. 5. In this case, some degree of flexibility is only present when the EHG operation line is within the

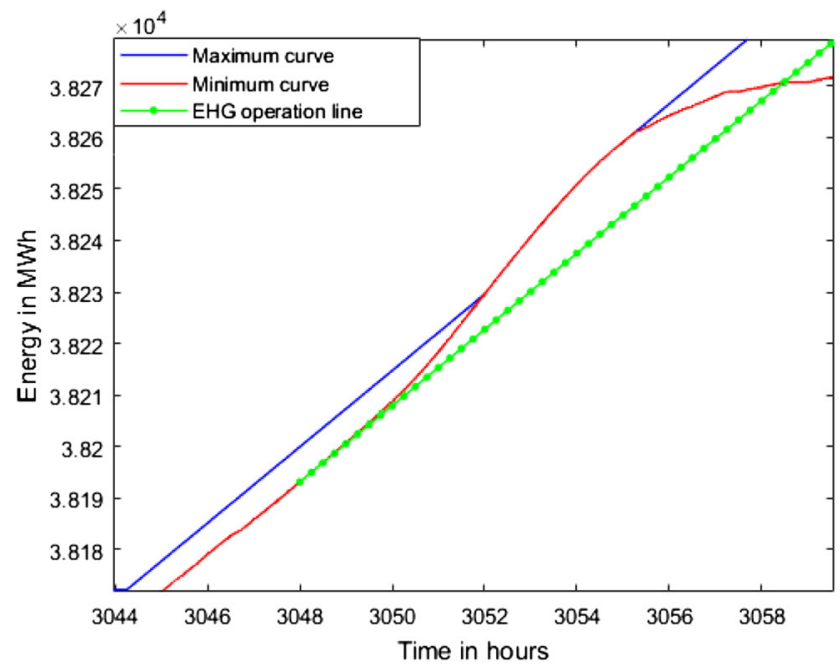

Fig. 4 No forced operation flexibility at $t=3048$ hours 


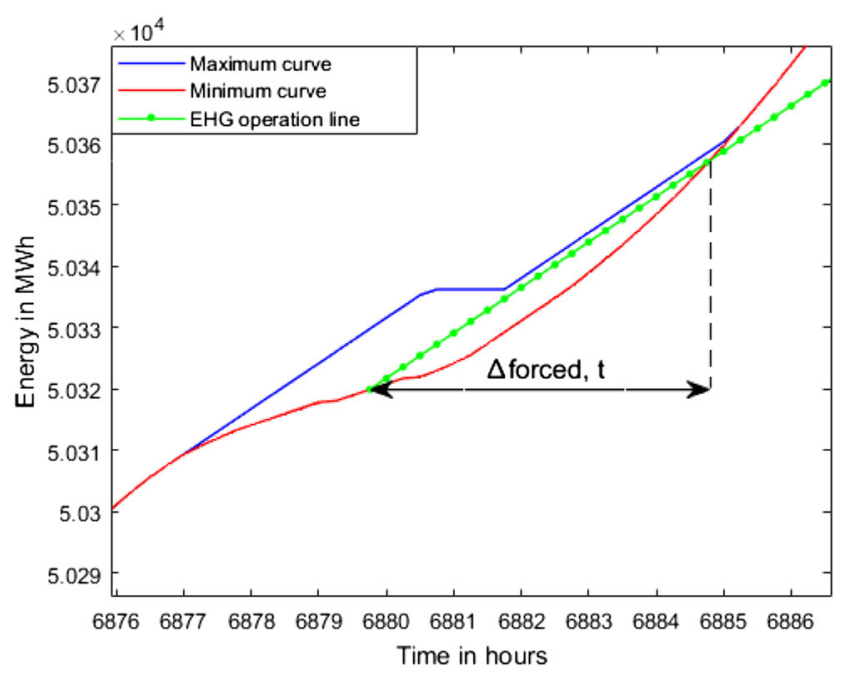

Fig. 5 Some forced operation flexibility at $t=6879.75$ hours

operational zone. Forced flexibility for these periods can be defined as the horizontal distance between $t$ and the time at which the linear EHG operation function starting in $\mathrm{Min}_{t}$ intersects with the Min curve again.

When the slope of EHG operation line is equal to the slope of the Min curve during some time period, as in the example of Fig. 6, all EHG units must be in operation for satisfying heat demand, which means that forced flexibility is zero.

\section{Deployable Power During Flexibility Periods}

As the deployment of several EHG units is considered in the method presented here, it also has to be analyzed what additional power can be forced to operate at each time step. There can be periods with some hours of forced

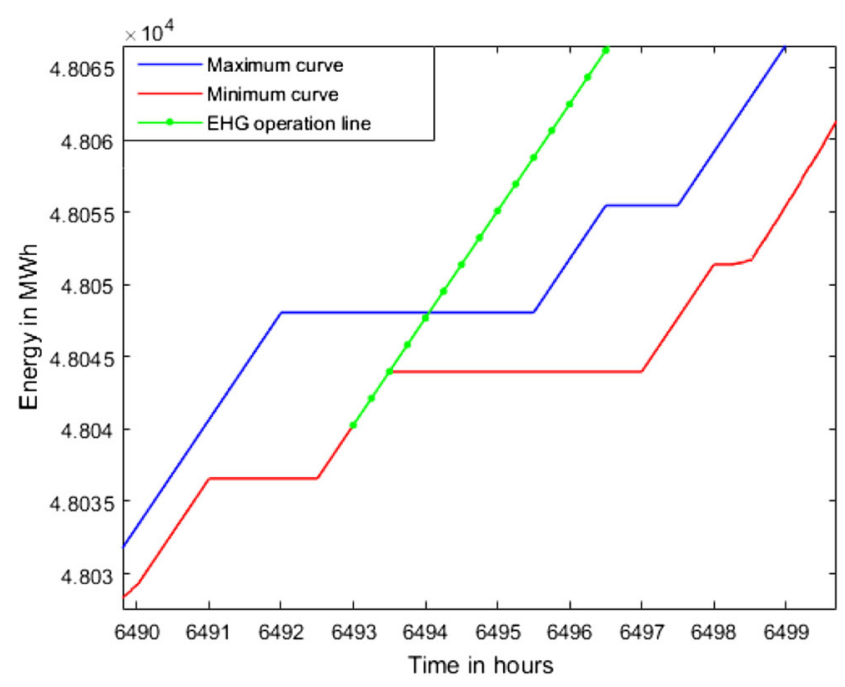

Fig. 6 No forced operation flexibility at $t=6493$ operation flexibility in which one or several unit(s) actually have to operate, while the operation of the remaining units is optional. An example for such a situation is given in Fig. 7. In this situation, one unit must operate under minimum operation conditions, and another three units may additionally operate.

Therefore, the information about the enforceable power $\Delta P_{\text {forced, } t}$ needs to be given as well for each time step, in order to correctly evaluate the flexibility situation. The power of the remaining units that are required to operate for satisfying demand are consequently not considered as being part of the flexible load (for HP) or generation (for CHP). The flexible power is easily calculated as the difference between the power available from all EHG units and the units operating in the minimum operation mode. This can be graphically interpreted as the difference in slope of the EHG operation line and the Min curve (see Fig. 7). As this difference can vary during the period of $\Delta_{\text {forced, } t}$, the average value is taken here as the metric for flexible power. This is given in Eq. 14.

$\Delta P_{\text {forced }, t}=\frac{1}{\Delta_{\text {forced }, t}} \sum_{\tau=1}^{\Delta_{\text {forced }, t}}\left(n_{t+\tau}^{\mathrm{Max}^{*}}-n_{t+\tau}^{\mathrm{Min} *}\right) \cdot \bar{P}_{\mathrm{EHG}}$

Depending on the requirements of the use case for the flexibility definition, more elaborated approaches for power definition could be used as an alternative for taking the arithmetic mean over the flexibility period.

The question of available power flexibility could also be asked inversely: How long can the operation of a given power (which must be a multiple of the single unit power $\left.\bar{P}_{\text {EHG }}\right)$ be delayed or forced without violating the heat demand and storage constraints? We should see that lower power can be forced over a longer time period than higher

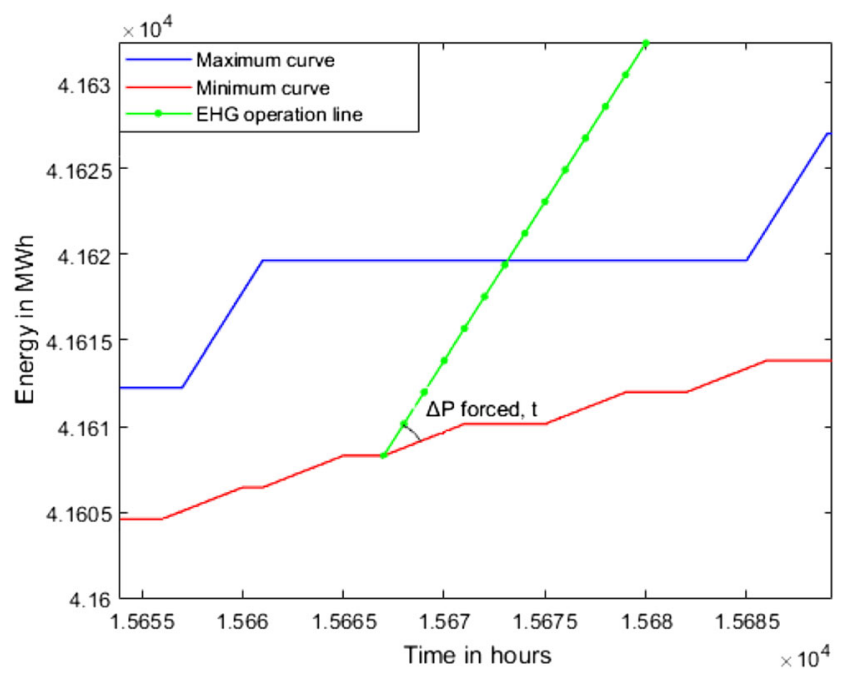

Fig. 7 Forced operation flexibility for part of available power at $t=$ 15667 
Fig. 8 Thermal load profile of a municipal district heating system in Germany in 2016

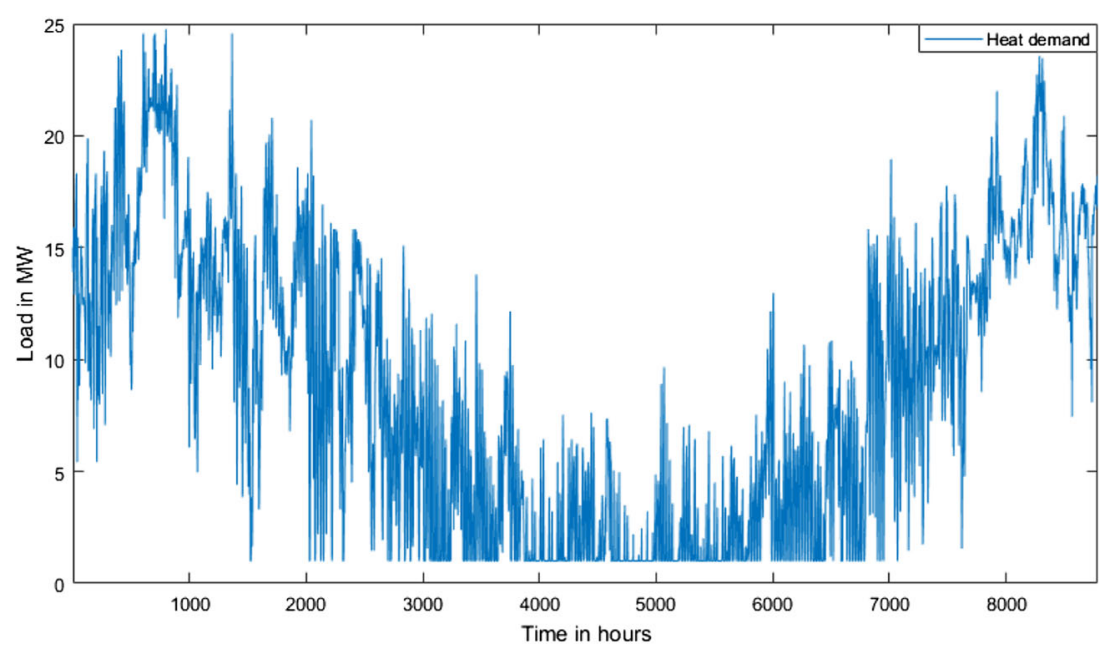

power. However, following the argument of Stinner et al. that high power flexibility for shorter time period is usually more useful than a limited amount of power, even if it is available over a longer time [27], this question is not further regarded here.

\section{Case Study and Results}

The described model has been applied to the case of a district heating (DH) system. The system set-up and the model results are presented in the following sections.

\section{System Description}

The DH system is equipped with several CHP units and operated by a municipal utility in Germany. In total, the heating system supplies energy to 4,000 consumers. Not all of the heat generators can be flexibly operated; for example, several wood gas CHP units always have priority and are operated whenever there is enough demand to use their heat output. Therefore, only the operation of the flexible
EHG units for serving the load not met by the "inflexible" units is considered. The load for flexible EHG subsystem varies between around $25 \mathrm{MW}$ during winter and around 4 MW during summer. The heat load data is aggregated to 15 minutes time intervals. The load profile of the year 2016 is depicted in Fig. 8. The peak load is observed in the month of January. It reaches a minimum in summer, where demand mainly comes from hot water usage.

There are four identical flexible CHP units of $\bar{P}_{\mathrm{EHG}}=$ 1.85 MW nominal thermal power (and 1.94 MW electrical power). Their minimum operation time and minimum down-time are both one hour, i. e. $d=r=4$. Several boilers are installed in the system, but as only the overall boiler power operating at time $t$ is relevant in the methodology applied here, they are all seen as one large unit with $\bar{P}_{\text {Boiler }}=26.65 \mathrm{MW}$. The thermal energy storage is a $4 \times 100 \mathrm{~m}^{3}$ water tank and allows a temperature range between 60 and $90{ }^{\circ} \mathrm{C}$. The energy capacity of this buffer storage is $\bar{E}_{\mathrm{TES}}=13.52 \mathrm{MWh}$.

With the given input data, the metrics introduced in "Methodology" have been calculated, as presented in the following subsections.
Fig. 9 Minimum operation mode of EHG units in a cluster with $k=4$

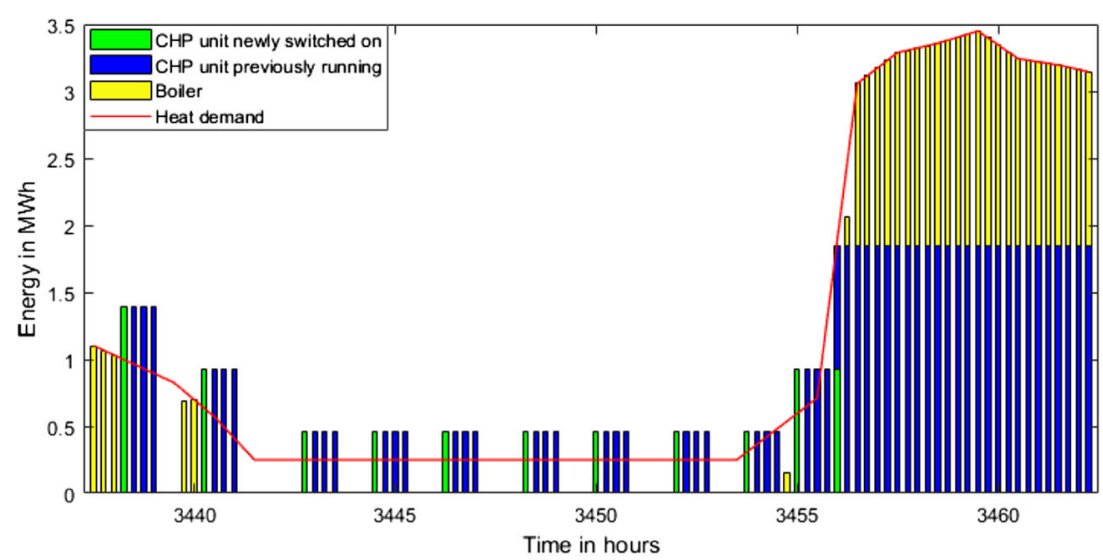


Fig. 10 Maximum operation mode of EHG units in a cluster with $k=4$

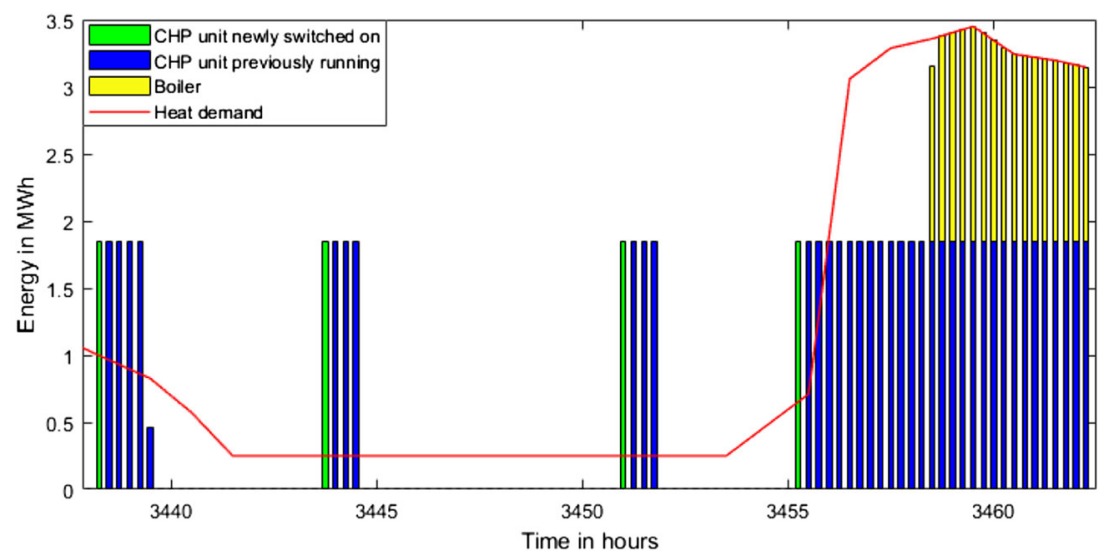

\section{CHP Unit Operation}

Figures 9 and 10 show the operation of the CHP units in the minimum and maximum operation mode during a given time period within the year. The heat demand is plotted as a solid line into the graphs. Demand decreases in the beginning of the observed period and then stays at a very low level, during which only one or no CHP unit operates in the minimum operation mode (one unit provides $0.4625 \mathrm{MWh}$ of heat in 15 minutes). In the maximum operation mode, all four generators are in operation at several times during the same period, and almost half of the produced thermal energy is stored. When demand increases towards the end of the period, all four CHP are running in both the modes, and the peak boiler steps in as soon as the buffer storage is depleted.

\section{Temporal Flexibility}

Figure 11 illustrates delayed flexibility, in hours, throughout the year 2016, displayed for each day of the year on the abscissa and each quarter of an hour within the day on the ordinate. It can be seen that most of the flexibility

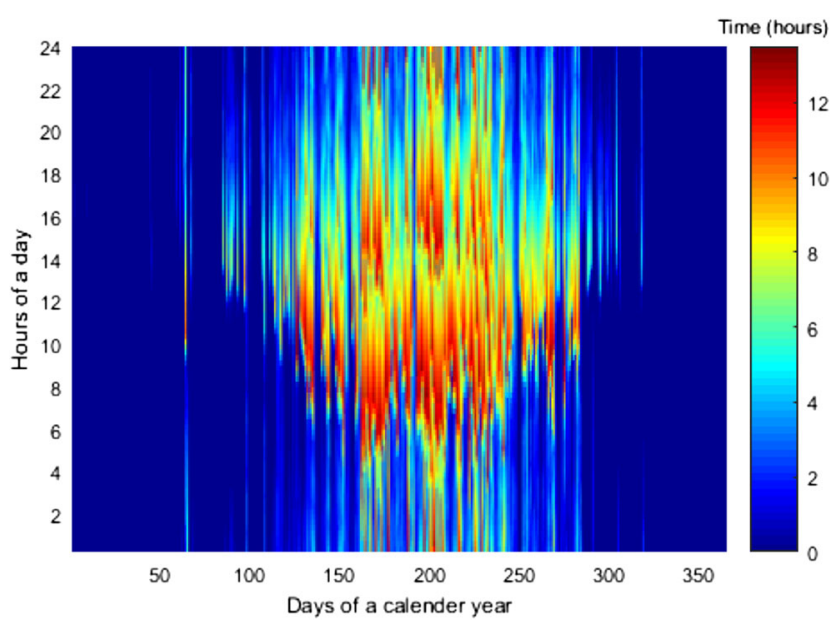

Fig. 11 Maximum available delayed flexibility in the CHP heating system (in hours) occurs during summer, particularly in the second half of the day. In contrast, and not surprisingly, delayed flexibility is often zero or very low during winter, as demand often exceeds CHP capacity during this season. Overall, there is a strong negative correlation between heat demand and delayed operation flexibility, which is also visible in the average daily flexibility value depicted in Fig. 11.

As demand is high at most times during winter, all CHP units are required to run, therefore also the forced operation flexibility is low, as it is shown in Fig. 12. Flexibility refers to a situation in which some operation can be requested on demand when it would otherwise not happen. The total amount of forced operation flexibility throughout the whole analyzed year ( $\sim 33 \mathrm{k}$ hours in total) is less than that of delayed flexibility ( $\sim 90 \mathrm{k}$ hours). ${ }^{1}$ Forced flexibility is highest during late spring and early autumn; during summer and winter, demand is either very low or very high, which both restricts the possibility of operating the CHP plants on demand, because the buffer fills quickly, or all units are required to satisfy the heat demand. The forced operation flexibility over the given year is shown in Fig. 12.

\section{Power Flexibility}

As discussed in "Deployable Power During Flexibility Periods", not all units can be flexibly deployed at each time during the forced operation flexibility time span. The average deployable power over the flexibility period calculated through Eq. 14 is displayed in Fig. 13. Possible values range between zero and $k \cdot \bar{P}_{\mathrm{EHG}}=7.4 \mathrm{MW}$.

During most of the times in which forced flexibility is available, the available power is in the order of $3.7 \mathrm{~kW}$. This corresponds to two or more out of four units being available for operation on demand. During the day and in the

\footnotetext{
${ }^{1}$ It has to be noted here that the informative value of the summed flexibility over a year is limited, as flexibility is multiply counted (most of the flexibility available in time $t$ is also available in $t+1$, and both values go into the overall sum). For comparison of the two flexibility types, or of different case studies, however, it is a useful indicator.
} 


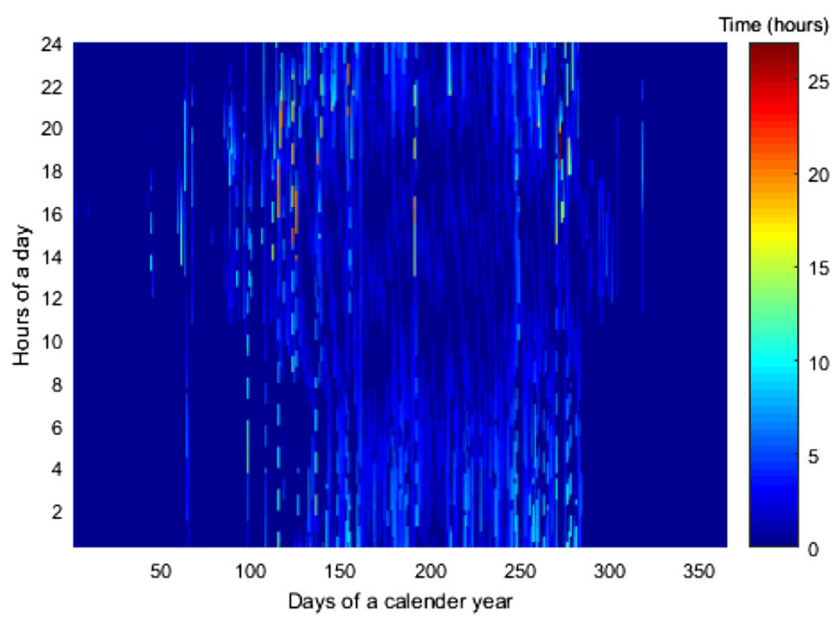

Fig. 12 Maximum available forced flexibility in the CHP heating system (in hours)

evening - especially in summer - it can often be observed that whenever there is forced flexibility, the available power is the equivalent of three or more units. Power flexibility is usually lower at night and in the early morning.

\section{Effects of Clustering and Operational Constraints}

For quantifying the effects of the number of units in an EHG cluster, as well as the operational constraints of minimum down-time and minimum operational time, the four following different cases are compared to each other:

1. Single unit, no constraints: The whole CHP power is provided by one single unit, and neither minimum down-time nor minimum operational time are considered $\rightarrow k=1, \bar{P}_{\mathrm{EHG}}=7.4 \mathrm{MW}, d=r=1$.

2. $\quad k$ units, no constraints: There are four identical CHP units, and also no operational constraints are considered $\rightarrow k=4, \bar{P}_{\mathrm{EHG}}=1.85 \mathrm{MW}, d=r=1$

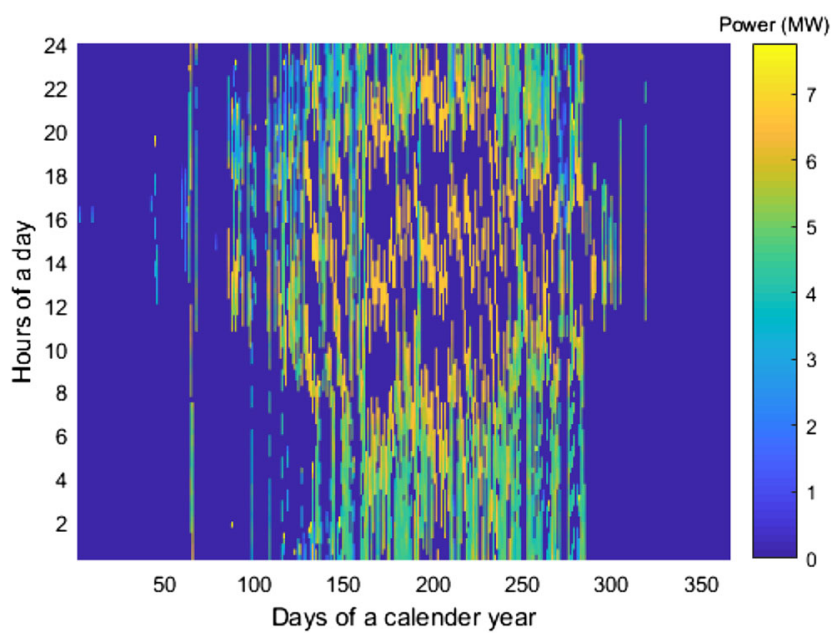

Fig. 13 Maximum available (electrical) power for forced flexibility (in MW)

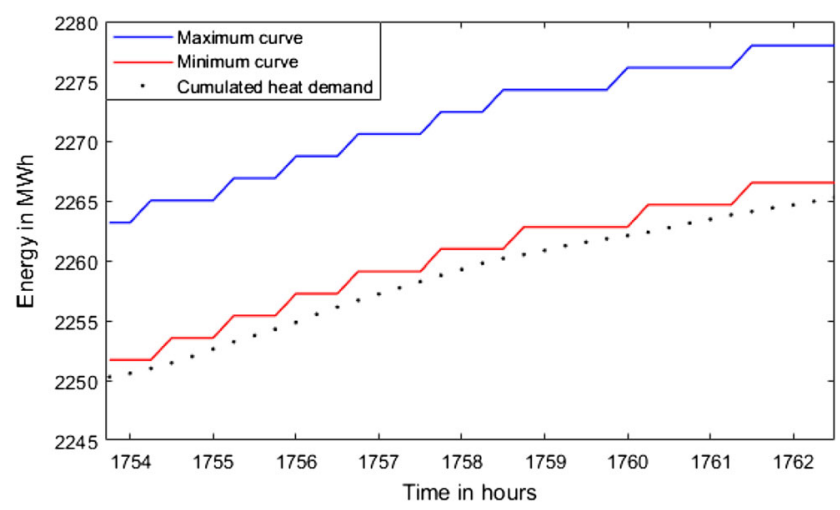

Fig. 14 Min \& Max curves and heat demand: single unit, no operational constraints

3. Single unit, with constraints: Operational constraints for both minimum down-time and minimum operational time are considered for one single CHP unit $\rightarrow$ $k=1, \bar{P}_{\mathrm{EHG}}=7.4 \mathrm{MW}, d=r=4$

4. $\quad k$ units, with constraints: There are four units with operational constraints $\rightarrow k=4, \bar{P}_{\mathrm{EHG}}=1.85 \mathrm{MW}$, $d=r=4$

Figures 14, 15, 16 and 17 shows the Min and Max curves for each of the four cases described above, and Figs. 18, 19, $20,21,22,23,24$ and 25 shows the carpet plots of delayed and forced flexibility. The comparison of the four cases reveals characteristic differences in CHP operation. Highest flexibility, represented by the widest operational zone, can be expected for a case without operational restrictions, and with the CHP power split into several units. The higher the number of units, the more opportunity there is to delay or force at least a subset of units, which provides more flexibility. This is confirmed by the simulation results observable in the given figures. With an average delayed operation flexibility of $3.13 \mathrm{~h}$ and forced operation flexibility of 1.57 , Case 2 offers the highest flexibility. Not surprisingly, the lowest flexibility is given for Case 3 with one unit and constraints $(1.98 \mathrm{~h}$ delayed and $0.37 \mathrm{~h}$ forced operation flexibility). The second highest average delayed

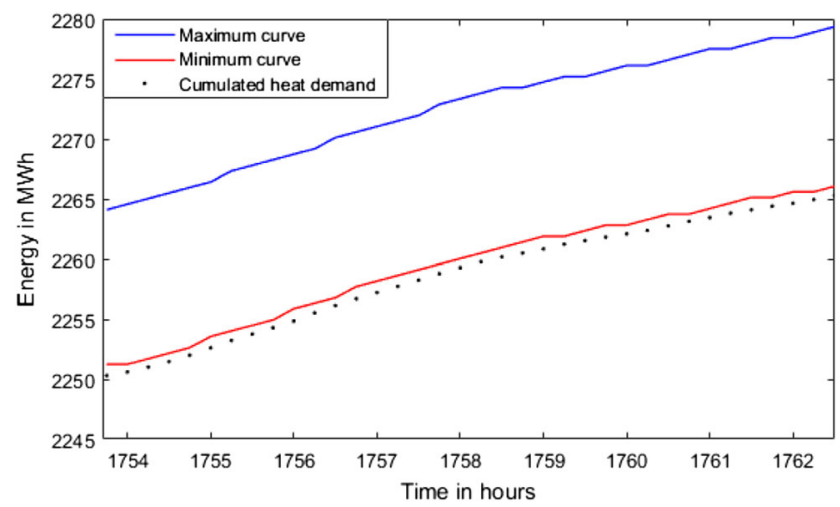

Fig. 15 Min \& Max curves and heat demand: $k=4$ units, no operational constraints 


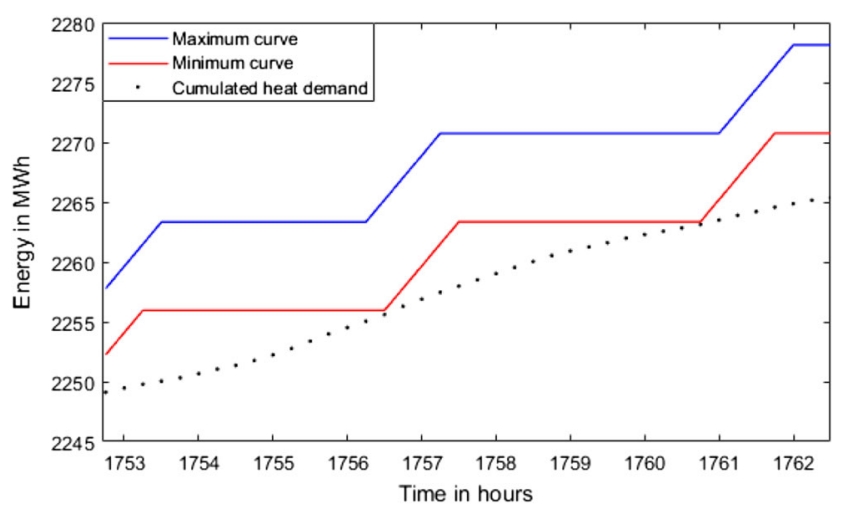

Fig. 16 Min \& Max curves and heat demand: single unit, operational constraints with $d=r=4$

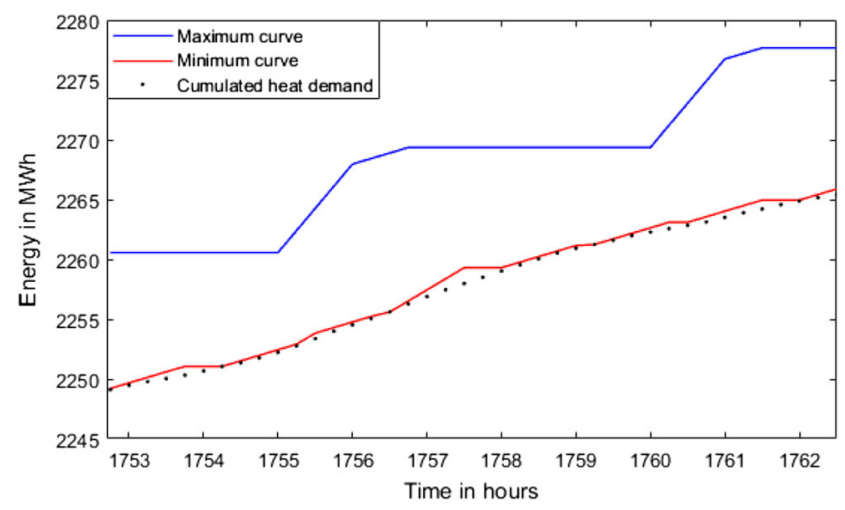

Fig. 17 Min \& Max curves and heat demand: $k=4$ units, operational constraints with $d=r=4$

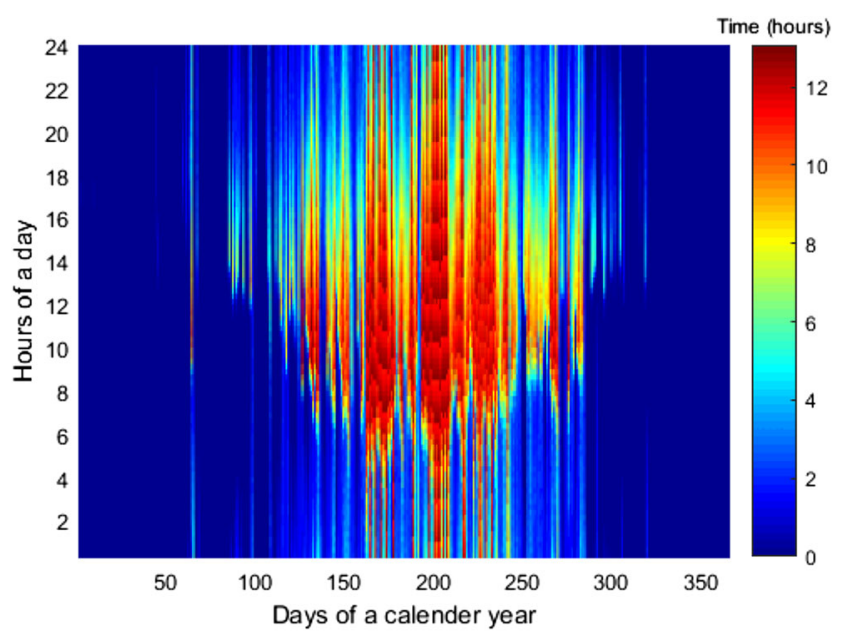

Fig. 18 Delayed operation flexibility: single unit, no operational constraints

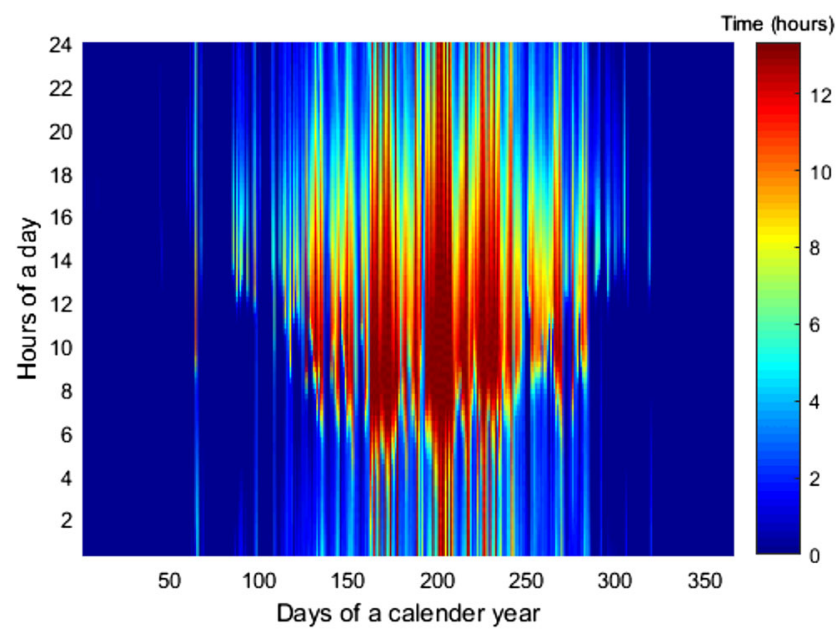

Fig. 19 Delayed operation flexibility: $k=4$ units, no operational constraints

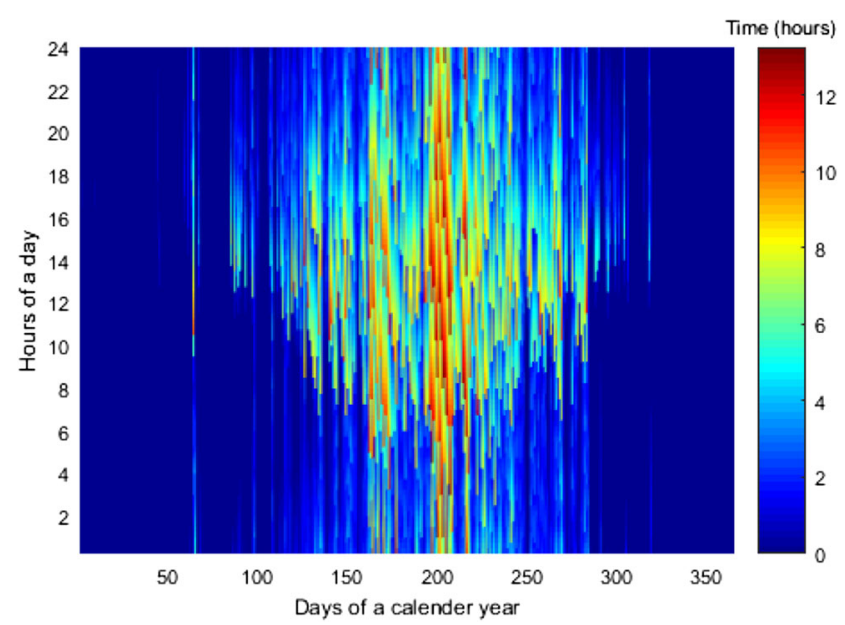

Fig. 20 Delayed operation flexibility: single unit, operational constraints with $d=r=4$

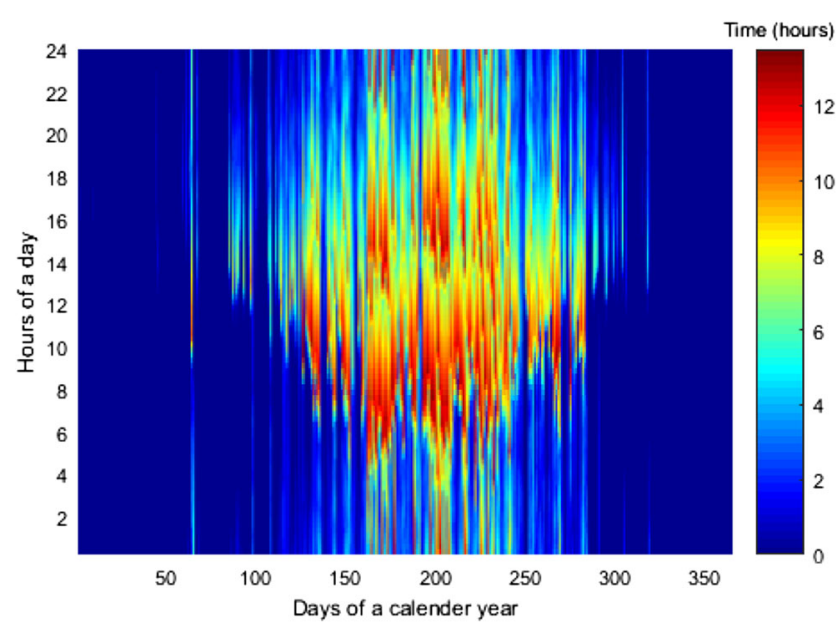

Fig. 21 Delayed operation flexibility: $k=4$ units, operational constraints with $d=r=4$ 


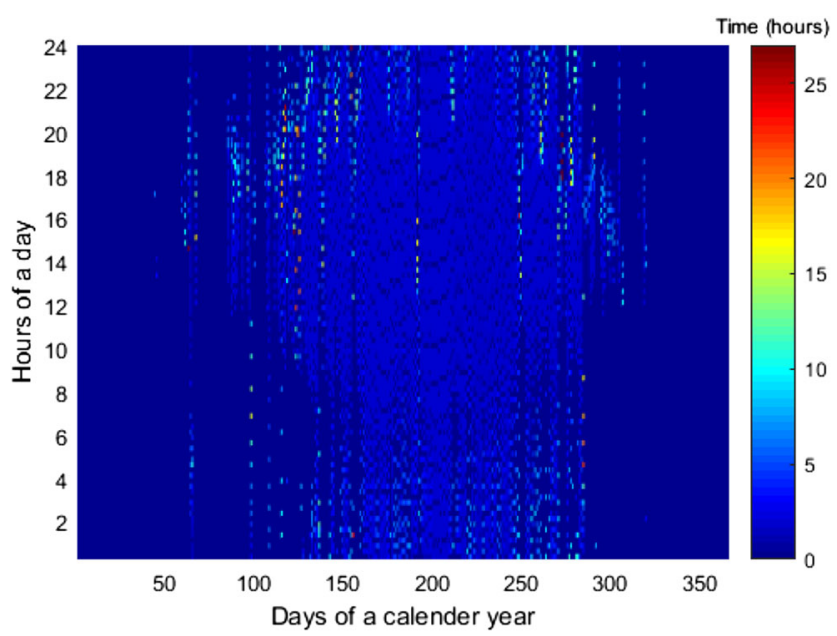

Fig. 22 Forced operation flexibility: single unit, no operational constraints

flexibility is given for Case 1 with $2.85 \mathrm{~h}$ (and then $2.57 \mathrm{~h}$ for Case 4). The operational constraints are, thus, limiting delayed flexibility much stronger than the restriction that only the whole capacity can be switched on or off in one block instead of more fine-grained switching with several units. Interestingly, in the case of forced operation flexibility, the order is the other way around: the second highest flexibility is given for Case $4(0.95 \mathrm{~h}$ ) (and then $0.74 \mathrm{~h}$ for Case 1). So, the limitation to only operate one unit decreases forced flexibility more than the operational constraints. This is the case, because the thermal storage is quickly filled when operating a large unit, so operation has to stop early. There are more times of forced flexibility for smaller units, although not always at the maximum power level.

Interestingly, operation in Case 4 reveals noticeable differences between the forms of the Min and Max curves. The possibility of step-wise modulation allows for staying closer to the cumulative heat demand in the Min case. In the

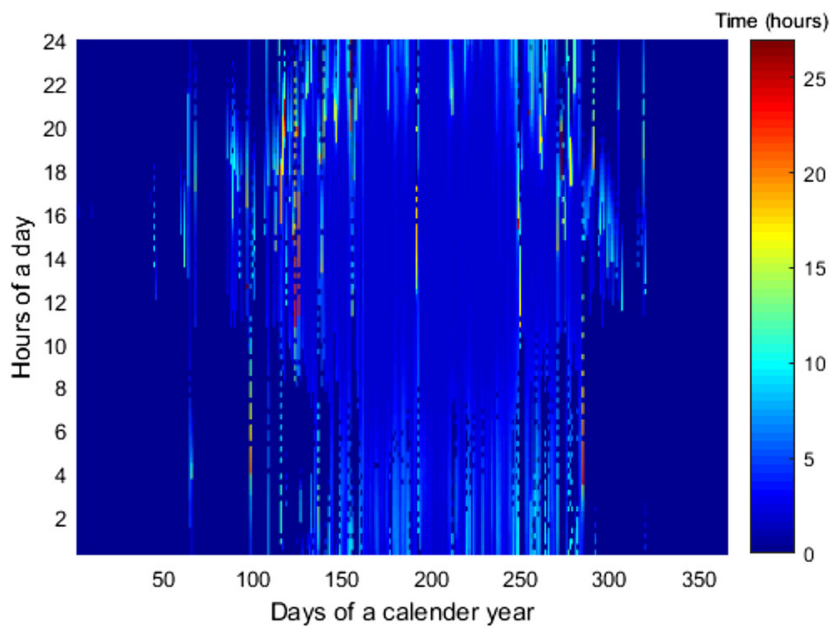

Fig. 23 Forced operation flexibility: $k=4$ units, no operational constraints

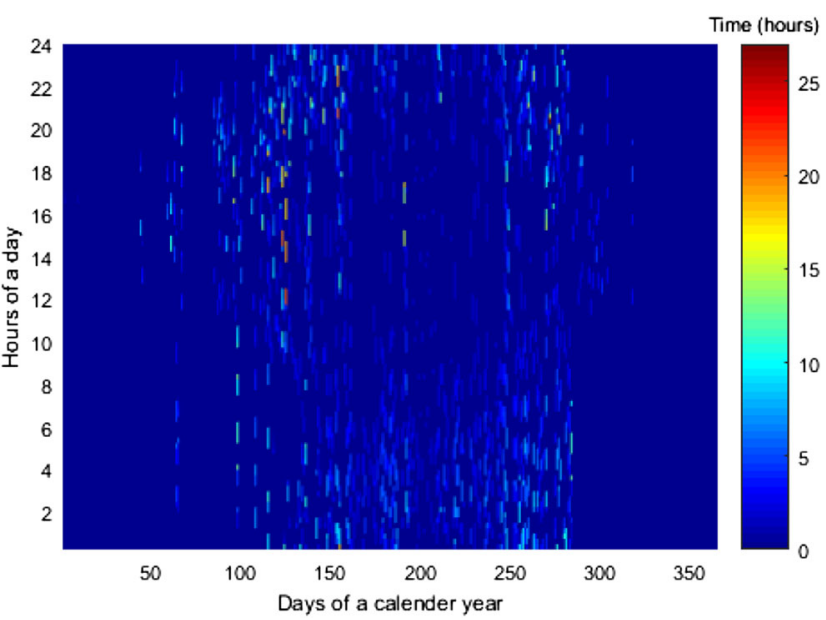

Fig. 24 Forced operation flexibility: single unit, operational constraints with $d=r=4$

Max mode, however, once there is free storage capacity and the units had been off for the required minimum operational time, as many units as possible switch on. Then, they often have to be switched off again after the minimum operational time, due to full storage. This behaviour results in several larger steps in the Max curve.

For assessing the effect of enlarging the buffer size, calculations for the reference case with $k=4, s=r=4$ have been done for several variations of TES capacity. It could be observed that whenever flexibility is more than zero hours, there is an almost linear relationship between delayed flexibility and buffer size. Doubling TES capacity results in a delayed flexibility that is twice as high as for the reference case. However, there are hardly more time intervals in which a positive delayed operation flexibility is observed, so delayed operation flexibility cannot be increased during winter. This is not surprising if it is kept in mind that demand exceeds CHP capacity during these times, so all units have to always operate throughout this season.

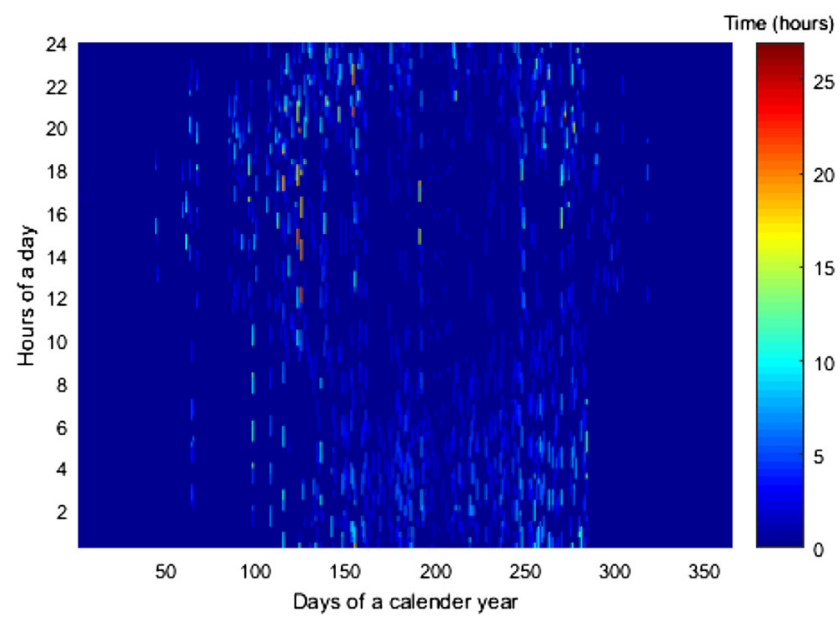

Fig. 25 Forced operation flexibility: $k=4$ units, operational constraints with $d=r=4$ 
For forced operation, a positive relationship between flexibility and buffer size can also be observed during summer and intermediate seasons. In periods of high heat demand, the buffer size has no effect on forced operation flexibility, as no excess heat is produced for storage.

\section{Discussion}

The case study reflects the flexibility of an EHG cluster which has not specifically been designed for flexibility delivery. This is a situation that is quite realistic for most EHG systems, because there are not many business opportunities for flexibility provision by small generation (or consumption) units, yet. If an EHG system should serve for flexibility purposes to a considerable extent, it is probably dimensioned differently, with larger generation capacity in relation to demand, and with larger storage capacity. Therefore, the potential seen here for the case study is rather at the lower end of the possible range. On the other hand, the currently chosen component sizes lead to high utilization of the CHP or HP systems, which is also important from an economical point of view. Flexibility potential and efficient use of generation capacities are therefore some conflicting goals which have to be considered simultaneously for economic system optimization.

Another factor that has not been considered here as a source for flexibility is the storage quality of the building itself. In the case study, calculations are based on the heat load, which in turn is a function of the heating system control, along with meteorological factors and user behavior. If a larger corridor of acceptable indoor temperatures was allowed for system operation, the thermal building characteristics could be included into the optimal operation of the system for providing more flexibility if needed.

The methodology presented here has also been applied to the case of another local heating grid in a community in Switzerland, which is supplied by a cluster of two large heat pumps. The power of the heat pumps is much larger in relation to the demand than in the CHP case, and the buffer is much smaller. This again corresponds to a common dimensioning of heat pump systems. In the model, however, situations were observed in which the Min curve exceeded the Max curve in some occasions, if minimum operational times and down-times are considered. In these situations, flexibility was considered as zero. The average delayed flexibility in that second case study was between 1.98 and $2.85 \mathrm{~h}$, depending on the cases considered (in analogy to cases 1 through 4 described here before).

The developed model quantifies the maximum available flexibility for each time interval. The actual flexibility available at a specific time, however, is dependent on the state that the system is in at this time, which is defined by the state of charge of the thermal energy storage unit, and by the actual operational time or down-time of each EHG unit at the given time instance. As such, the calculated flexibility constitutes an upper limit, which might not be met in actual operation of the plant. This is a limitation of the chosen approach. However, as some flexibility requirements can be reliably forecast, a sufficiently long lead time enables the system to move into the necessary state for the maximum (or desired) flexibility potential. This implies that the operational flexibility is reduced or zero during this lead time. Therefore, the flexibility potential quantified by the method proposed here is especially useable for a flexibility demand that is foreseen well in advance.

To make the model useful for shorter-term flexibility demand, it would be a valuable extension to provide the flexibility information in the form of a probability density function, assuming some realistic probability function for the reference operation of the system. An alternative could be to calculate the reference schedule based on some optimization criterion, and to calculate only the remaining flexibility based on this reference. Another possible extension is to quantify the flexibility as a function of the lead time between a flexibility request and its activation. Finally, the model proposed here assumes perfect forecast of the heat demand. Stochastic approaches that explicitly model uncertainty could add valuable insights to the approach presented here. These extensions could be paths for future research.

\section{Conclusion}

A model that calculates the flexibility potential provided by a cluster of small-scale electricity-coupled heat generators with thermal energy storage has been developed. An operational zone in which EHG operation is possible has been defined. This operational zone allows to quantify possible paths of operation, and reveals how long EHG operation can be forced or delayed, thus delivering positive (negative) or negative (positive) flexibility to the power system when operating a cluster of CHP (HP) units. Clusters of $k$ EHG units can be modeled, and minimum operation time and minimum down-time of the heat generators are accounted for. These characteristics are limiting the available flexibility in practical applications, and must therefore be taken into account in a realistic EGH cluster model.

The model has been implemented and run with heat load data of a local heat network operated in Germany in 2016. As expected, delayed flexibility peaks during summer, when heat demand is lowest. Hardly any flexibility is available during winter. Forced flexibility, on the other hand, peaks during spring and autumn, at intermediate heat demand levels, and is also hardly available in winter. 
Further analyses have been carried out to determine what factors affect the flexibility most. Splitting the EHG capacity into multiple smaller instead of one larger unit, thus allowing for some degree of modulation, does not increase the delayed operation flexibility so much (e. g. $10 \%$ more for $k=4$ units in comparison to one unit). However, it more than doubles the average forced operation flexibility (although often at reduced power). Considering minimum operational time and minimum down-time of the EHG units considerably reduces both the delayed and forced operation flexibility, especially in the case of one large unit (e. g. $30 \%$ less delayed and 50\% less forced flexibility for $s=r=4$ than without operational constraints in case of $k=1$ unit, and $18 \% / 40 \%$ delayed / forced flexibility less for $k=4$ ).
The size of the buffer has a very strong positive correlation with delayed operational flexibility during summer, and a less pronounced positive correlation with the forced flexibility; however, increasing the buffer size does not increase the flexibility of the system during winter. As the heat generators operate during most time intervals in winter, there is no flexibility in operation, neither delayed nor forced.

Acknowledgements The research described in this article was carried out in the framework of the projects mikroVKK-Demonstration der Machbarkeit, Wirtschaftlichkeit und der Smart-Grid-Potentiale von Virtuellen Kraftwerken mit Mikro- und Mini-BHKW (State of BadenWürttemberg, grant BWSG15003), and C/sells - Das Energiesystem der Zukunft im Sonnenbogen Süddeutschlands (Federal Ministry of Economic Affairs and Energy, grant 03SIN103).

\section{Appendix}

Fig. 26 Exemplary flowchart visualizing the calculation of $n_{t}$ as a basis for the Min curve; for simplicity, $n_{t}$ is also used for expressing $n_{t}^{*}$, and superscript Min is omitted

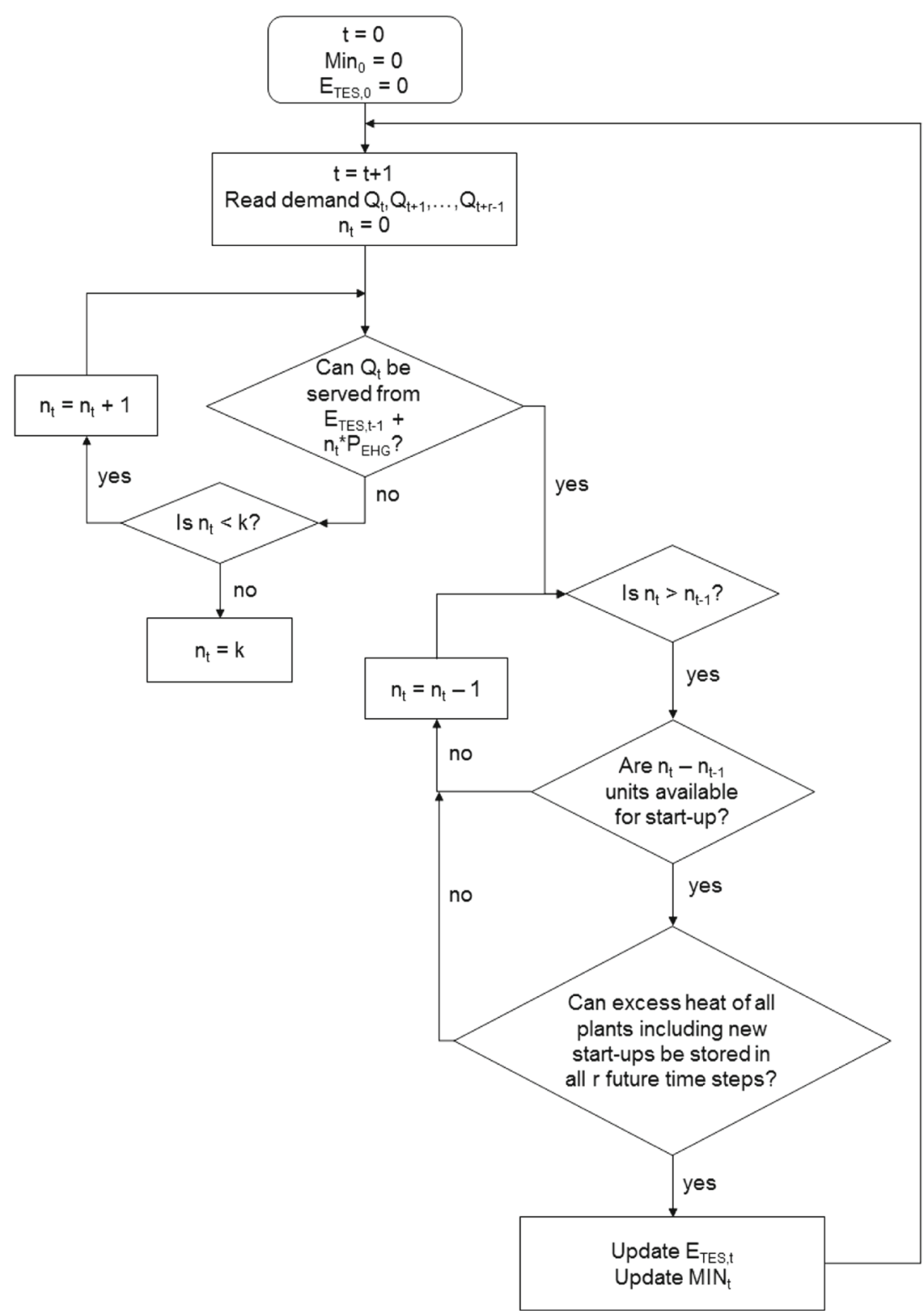




\section{References}

1. Alizadeh MI, Parsa Moghaddam M, Amjady N, Siano P, SheikhEl-Eslami MK (2016) Flexibility in future power systems with high renewable penetration: a review. Renew Sustain Energy Rev 57:1186-1193

2. Bolívar Jaramillo L, Weidlich A (2016) Optimal microgrid scheduling with peak load reduction involving an electrolyzer and flexible loads. Appl Energy 169:857-865. https://doi.org/10.1016/ j.apenergy.2016.02.096

3. Schulz C, Roder G, Kurrat M (2005) Virtual power plants with combined heat and power micro-units. In: International conference on future power systems (FPS), pp 1-5. Amsterdam, The Netherlands

4. Chauhan K, Chauhan RK (2017) Optimization of grid energy using demand and source side management for DC microgrid. J Renewable Sustainable Energy 9(035101):1-15. https://doi.org/ 10.1063/1.4984619

5. Cochran J, Miller M, Zinaman O, Milligan M, Arent D, Palmintier B, O’Malley M, Mueller S, Lannoye E, Tuohy A et al (2014) Flexibility in 21st century power systems. Tech. rep., National Renewable Energy Laboratory (NREL), Golden CO

6. Coninck RD, Helsen L (2016) Quantification of flexibility in buildings by cost curves - methodology and application. Appl Energy 162:653-665. https://doi.org/10.1016/j.apenergy.2015.10. 114. http://www.sciencedirect.com/science/article/pii/S03062619 15013501

7. D'Hulst R, Labeeuw W, Beusen B, Claessens S, Deconinck G, Vanthournout K (2015) Demand response flexibility and flexibility potential of residential smart appliances: Experiences from large pilot test in belgium. Appl Energy 155(Supplement C):79-90. https://doi.org/10.1016/j.apenergy.2015.05.101

8. Dimeas A, Drenkard S, Hatziargyriou N, Karnouskos S, Kok K, Ringelstein J, Weidlich A (2014) Smart houses in the smart grid: developing an interactive network. IEEE Electrification Magazine 2(1):81-93. https://doi.org/10.1109/MELE.2013.2297032

9. Eid C, Codani P, Perez Y, Reneses J, Hakvoort R (2016) Managing electric flexibility from distributed energy resources: a review of incentives for market design. Renew Sust Energ Rev 64:237-247. https://doi.org/10.1016/j.rser.2016.06.008. http:// www.sciencedirect.com/science/article/pii/S1364032116302222

10. Finck C, Li R, Kramer R, Zeiler W (2018) Quantifying demand flexibility of power-to-heat and thermal energy storage in the control of building heating systems. Appl Energy 209:409-425. https://doi.org/10.1016/j.apenergy.2017.11.036

11. Fischer D, Wolf T, Wapler J, Hollinger R, Madani H (2017) Model-based flexibility assessment of a residential heat pump pool. Energy 118:853-864. https://doi.org/10.1016/j.energy.2016. 10.111

12. Förderer K, Ahrens M, Bao K (2018) Modeling flexibility using artificial neural networks. Energy Informatics 1(Suppl 1, 21):7391

13. Gils HC (2014) Assessment of the theoretical demand response potential in Europe. Energy 67:1-18. https://doi.org/10.1016/j. energy.2014.02.019

14. Kohlhepp P, Hagenmeyer V (2017) Technical potential of buildings in germany as flexible power-to-heat storage for smart-grid operation. Energ Technol 5:1084-1104. https://doi.org/10.1002/ ente. 201600655

15. Kondziella H, Bruckner T (2016) Flexibility requirements of renewable energy based electricity systems - A review of research results and methodologies. Renew Sustain Energy Rev 53:10-22

16. Lopes RA, Chambel A, Neves J, Aelenei D, Martins J (2016) A Literature Review of Methodologies Used to Assess the
Energy Flexibility of Buildings. Energy Procedia 91:1053-1058. https://doi.org/10.1016/j.egypro.2016.06.274

17. Ma J, Silva V, Belhomme R, Kirschen DS, Ochoa LF (2013) Evaluating and planning flexibility in sustainable power systems. IEEE Trans Sustainable Energy 4(1):200-209

18. MacDougall P, Kosek AM, Bindner H, Deconinck G (2016) Applying machine learning techniques for forecasting flexibility of virtual power plants. In: 2016 IEEE electrical power and energy conference (EPEC), pp 1-6. https://doi.org/10.1109/EPEC.2016. 7771738

19. Nosair H, Bouffard F (2015) Flexibility envelopes for power system operational planning. IEEE Trans Sustainable Energy 6(3):800-809

20. Nuytten T, Claessens B, Paredis K, van Bael J, Six D (2013) Flexibility of a combined heat and power system with thermal energy storage for district heating. Appl Energy 104:583-591. https://doi.org/10.1016/j.apenergy.2012.11.029

21. Pedersen TS, Nielsen KM, Andersen P (2014) Maximizing storage flexibility in an aggregated heat pump portfolio. In: 2014 IEEE conference on control applications (CCA), pp 286-291. https://doi.org/10.1109/CCA.2014.6981360

22. Reynders G, Diriken J, Saelens D (2017) Generic characterization method for energy flexibility: applied to structural thermal storage in residential buildings. Appl Energy 198:192-202. https://doi.org/10.1016/j.apenergy.2017.04.061

23. Reynders G, Lopes RA, Marszal-Pomianowska A, Aelenei D, Martins J, Saelens D (2018) Energy flexible buildings: An evaluation of definitions and quantification methodologies applied to thermal storage. Energy Build 166:372390. https://doi.org/10.1016/j.enbuild.2018.02.040. http://www. sciencedirect.com/science/article/pii/S037877881732947X

24. Roossien B, Hommelberg M, Warmer C, Kok K, Turkstra JW (2008) Virtual power plant field experiment using 10 micro-CHP units at consumer premises. In: CIRED seminar 2008: Smart grids for distribution, pp 1-4. https://doi.org/10.1049/ic:20080410

25. Shoreh MH, Siano P, Shafie-khah M, Loia V, Catalão JP (2016) A survey of industrial applications of Demand Response. Electr Power Syst Res 141:31-49. https://doi.org/10.1016/j.epsr.2016.07. 008

26. Six D, Desmedt J, van Bael J, Vanhoudt D (2011) Exploring the flexibility potential of residential heat pumps combined with thermal energy storage for smart grids. In: 21st international conference on electricity distribution CIRED, pp 6-9

27. Stinner S, Huchtemann K, Müller D (2016) Quantifying the operational flexibility of building energy systems with thermal energy storages. Appl Energy 181:140-154. https://doi.org/10.1016/j. apenergy.2016.08.055

28. Stram BN (2016) Key challenges to expanding renewable energy. Energy Policy 96(Supplement C):728-734. https://doi.org/10. 1016/j.enpol.2016.05.034

29. Ulbig A, Andersson G (2015) Analyzing operational flexibility of electric power systems. Int J Electr Power Energy Syst 72:155164. https://doi.org/10.1016/j.ijepes.2015.02.028

30. Verdolini E, Vona F, Popp D (2016) Bridging the gap: Do fast reacting fossil technologies facilitate renewable energy diffusion? Nota di Lavoro 51.2016 mitigation, innovation and transformation pathways, Fondazione Eni Enrico Mattei, Milan, Italy

31. Zadé $\mathrm{M}$, Incedag $\mathrm{Y}$, El-Baz $\mathrm{W}$, Tzscheutschler $\mathrm{P}$, Wagner U (2018) Prosumer integration in flexibility markets: A bid development and pricing model. In: IEEE conference on energy internet and energy system integration. IEEE, Peking

Publisher's Note Springer Nature remains neutral with regard to jurisdictional claims in published maps and institutional affiliations. 